Supporting Information for the article:

\title{
Graphene tunable transparency to tunneling electrons: A direct tool to measure the local coupling.
}

Héctor González-Herrero ${ }^{1}$, Pablo Pou ${ }^{2,3}$ Jorge Lobo-Checa ${ }^{4}$, Delia Fernández-Torre ${ }^{2}$, Fabian Craes ${ }^{6}$, Antonio J. Martínez-Galera ${ }^{1,5}$, Miguel M. Ugeda ${ }^{1}$, Martina Corso ${ }^{4,6}$, J. Enrique Ortega 4,7,8 , José M. Gómez-Rodríguez ${ }^{1,3}$, Rubén Pérez ${ }^{2,3}$, Iván Brihuega ${ }^{1,3^{*}}$.

AUTHOR ADDRESS.

${ }^{1}$ Departamento Física de la Materia Condensada, Universidad Autónoma de Madrid, E-28049

Madrid, Spain.

${ }^{2}$ Departamento Física de la Materia Condensada, Universidad Autónoma de Madrid, E-28049

Madrid, Spain.

${ }^{3}$ Condensed Matter Physics Center (IFIMAC), Universidad Autónoma de Madrid, E-28049 Madrid, Spain.

${ }^{4}$ Centro de Física de Materiales (CSIC-UPV-EHU) and Materials Physics Center (MPC), Manuel Lardizábal 5, E-20018 San Sebastián, Spain

${ }^{5}$ II. Physikalisches Institut, Universität zu Köln, Zülpicher Straße 77,50937 Köln, Germany.

${ }^{6}$ CIC nanoGUNE, 20018 Donostia-San Sebastian, Spain.

${ }^{7}$ Ikerbasque, Basque Foundation for Science, 48011 Bilbao, Spain.

${ }^{8}$ Donostia International Physics Center DIPC, E-20018 San Sebastián, Spain

${ }^{9}$ Departamento de Física Aplicada I, Universidad del País Vasco, E-20018 San Sebastián, Spain

*) Corresponding author: ivan.brihuega@uam.es

KEYWORDS.

Graphene, $\mathrm{Cu}(111)$, electronic properties, STM, DFT, ARPES. 


\section{STM characterization of the general morphology of the graphene/Cu(111) samples.}

We have grown our graphene/Cu(111) samples using the ethylene irradiation method developed by some of us ${ }^{1}$. Our STM images show large regions covered by high quality graphene flakes (Figure S1a), where we can observe different moiré patterns depending on the relative rotation of the graphene sheet with respect to the $\mathrm{Cu}(111)$ substrate (Figures S1b,c,d).

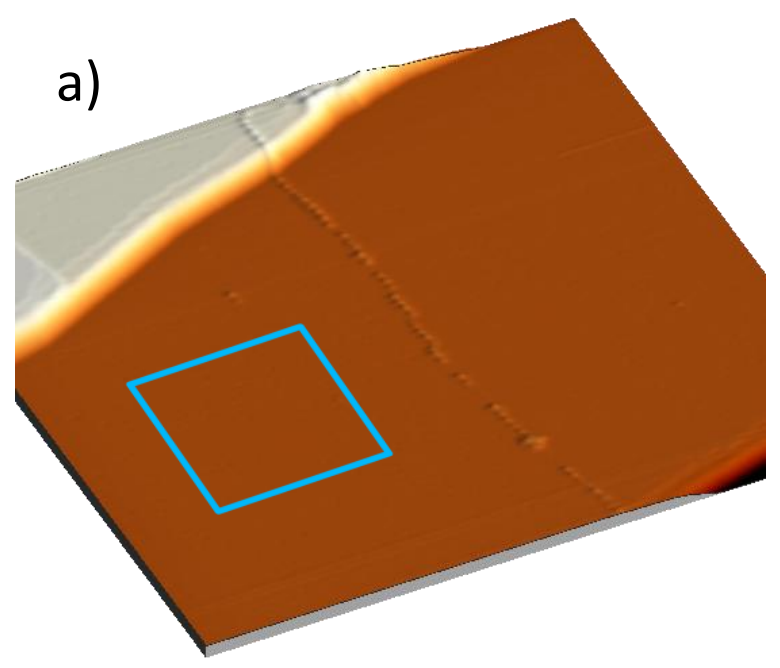

b)

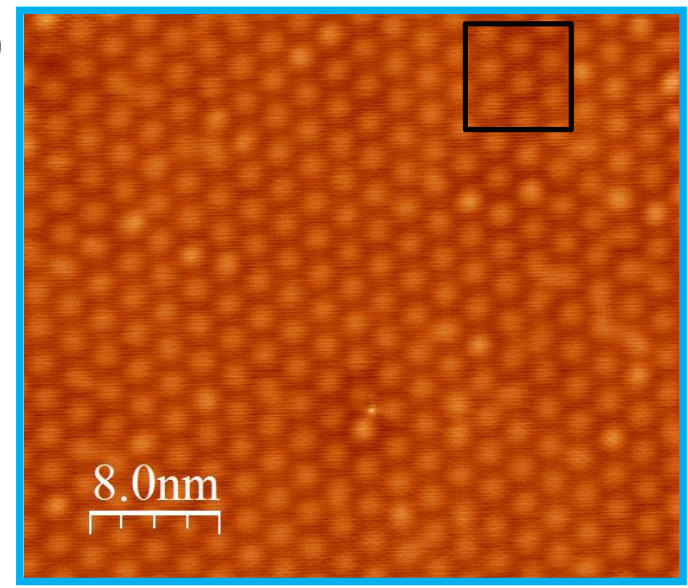

c)
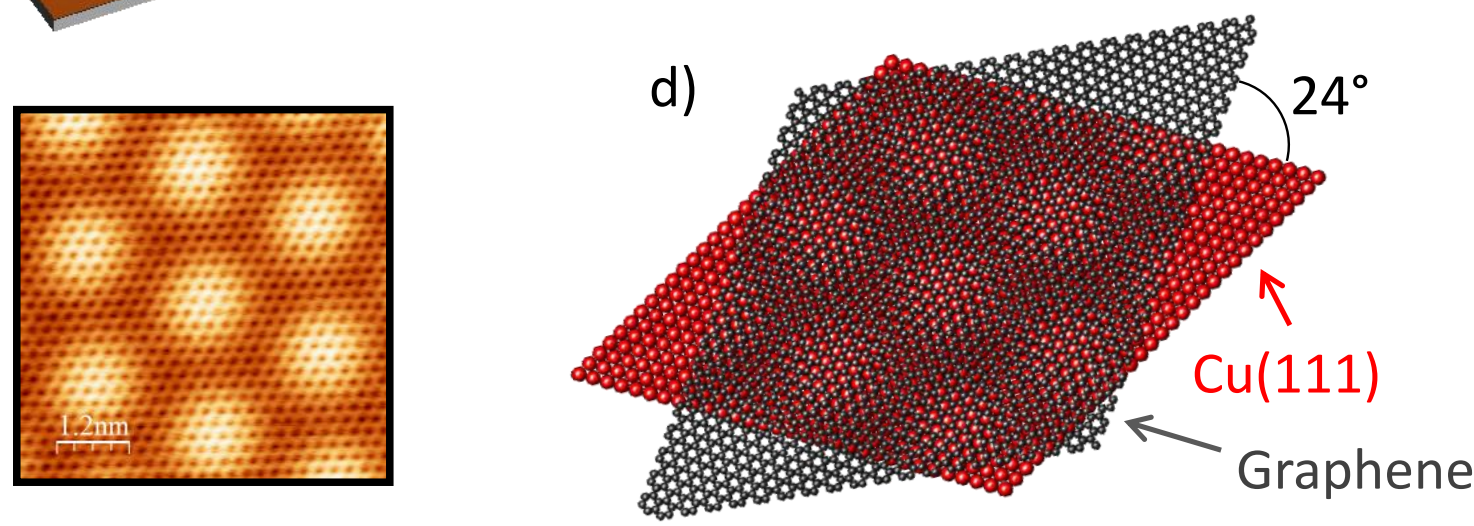

FigureS1. STM characterization of the general morphology of G/Cu(111) samples: a) 140x140 $\mathrm{nm}^{2}$ region of graphene b) $40 \times 35 \mathrm{~nm}^{2}$ zoom of Figure S1a where we can observe a moiré superstructure. c) $6 \times 6 \mathrm{~nm}^{2}$ zoom of previous image where atomic resolution on graphene can be observed. d) Schematic model of the moire shown in Figure S1c where a graphene lattice (in black) is superposed onto the $\mathrm{Cu}(111)$ lattice (in red) with a rotation of $24^{\circ}$ 
a)
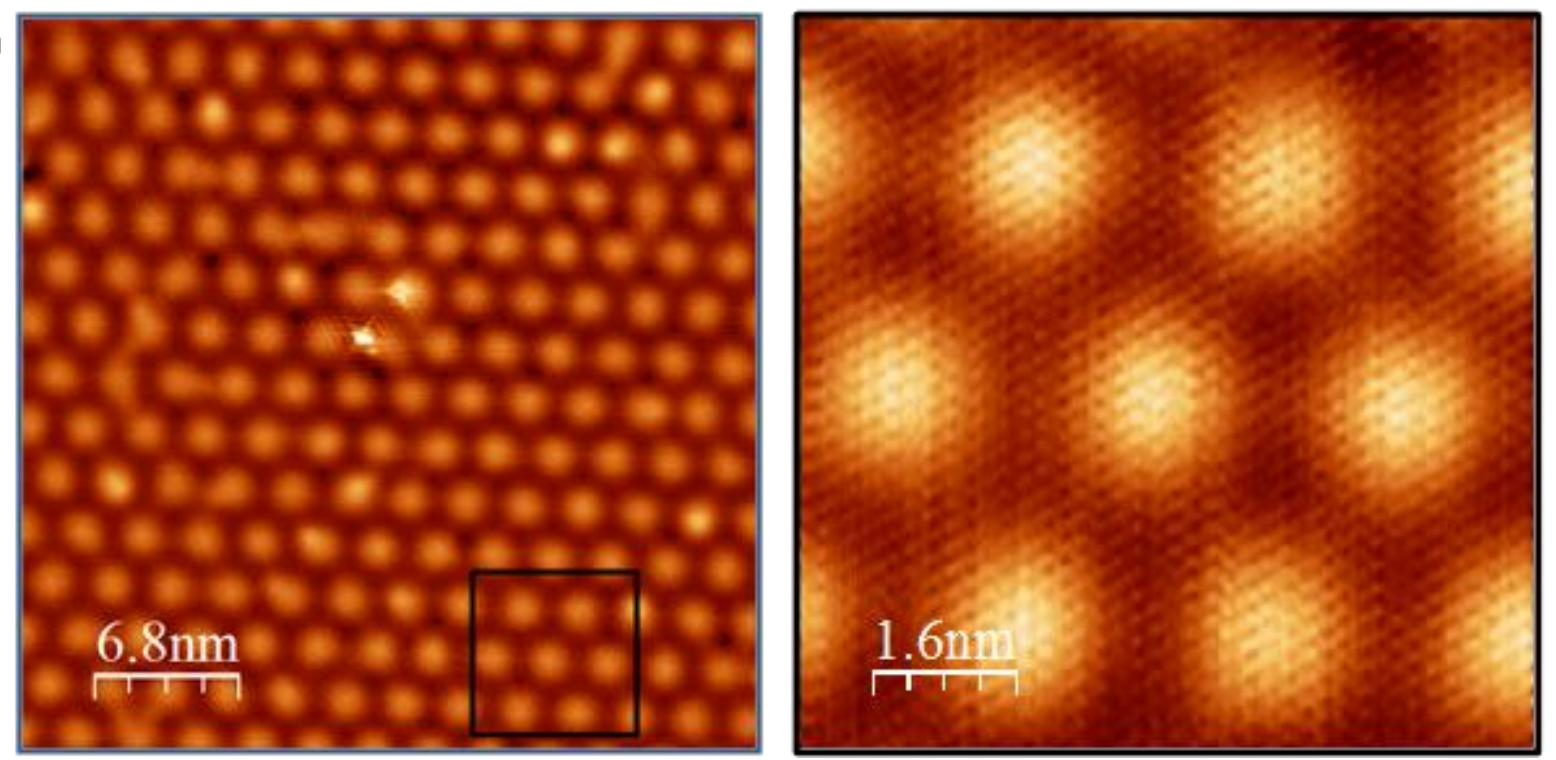

FigureS2. Atomic scale resolution of Figure 1a: a) Same $34 x 34 \mathrm{~nm}^{2}$; STM image as in Figure 1a of the mane manuscript. Sample bias: $50 \mathrm{meV}$; tunnelling current: $0.4 n A$. b) $8 \times 8 \mathrm{~nm}^{2}$ zoom of in of the region outlined by a black square in image a) where atomic resolution on graphene can be observed. Sample bias: $100 \mathrm{meV}$; tunnelling current: $0.4 n \mathrm{~A}$ The moiré pattern originates from the existing rotation of $25^{\circ}$ between the graphene layer and the $\mathrm{Cu}(111)$. 


\section{STM characterization of $2 \mathrm{D}$ electronic states.}

Of particular interest for the present study is the characterization of 2D electronic states by STM. Pioneering STM works showed the possibility to visualize the elastic scattering of quasiparticles from these $2 \mathrm{D}$ electronic states with the different impurities of the system in the form of quantum interference patterns ${ }^{2,3}$. The interference between incident and scattered 2D quasiparticles gives rise to LDOS spatial modulations related to the particular symmetry of the $2 \mathrm{D}$ constant-energy contour of the system (2D constant-energy contours present, in the reciprocal space, all the available electronic states for each energy). In a very simple picture, elastic scattering at impurities couple different states $\vec{k}(E)$ and $\vec{k}^{\prime}(E)$ of the constant-energy contour, being more probable the scattering processes corresponding to good nesting vectors. It was also shown ${ }^{4,5}$ that the $2 \mathrm{D}$ Fourier Transform (2D-FT) of STM $d I / d V$ images is closely related to a map of the constant-energy contour of the $2 \mathrm{D}$ state since it reflects the possible scattering vectors of the system, being this an efficient method to directly obtain $k(E)$.

One of the first systems where this was demonstrated was the $\mathrm{Cu}(111)$ surface ${ }^{2}$. Pristine $\mathrm{Cu}(111)$ presents a Shockley surface state characterized by a 2D free-electron like behaviour with an isotropic parabolic band dispersion given by: $E=E_{0}+\hbar^{2} k^{2} / 2 m^{*}$, where $E_{0}$ is the band edge and $m^{*}$ is the effective mass, see Figure S3a. The 2D constant-energy contours correspond then to a ring of radius $k(E)$ centred at the $\Gamma$ point of the Brillouin zone, see right panel in Figure S3a. Accordingly, as shown in Figure S3b, the 2D-FT of $d I / d V$ maps, measured at energy $E=e \cdot V$, show a central ring of radius $2 k(E)$ (here scattering between states $\vec{k}(E)$ and $-\vec{k}(E)$, with a scattering vector $2 \vec{k}(E)$, are the most efficient processes as indicated in the right panel of Figure $3 b$. The energy dispersion $k(E)$ of the clean $\mathrm{Cu}(111)$ surface is then obtained by performing the 2D-FT of $d I / d V$ maps measured at different energies Figures S3c-g. The band edge can be determined from the energy position of the sudden step observed in $d I / d V$ curves measured on clean terraces Figure $S 3 h^{6}$.We obtained $E_{0}=-430 \mathrm{meV}$ and $m^{*}=0.390 m_{e}$ ( $m_{e}$ is the free-electron mass) for the $\mathrm{Cu}(111)$ surface state before graphene formation, in perfect agreement with the values reported in the literature ${ }^{2,4,7}$, see Figure S3i. 


\section{$\mathrm{Cu}(111)$}
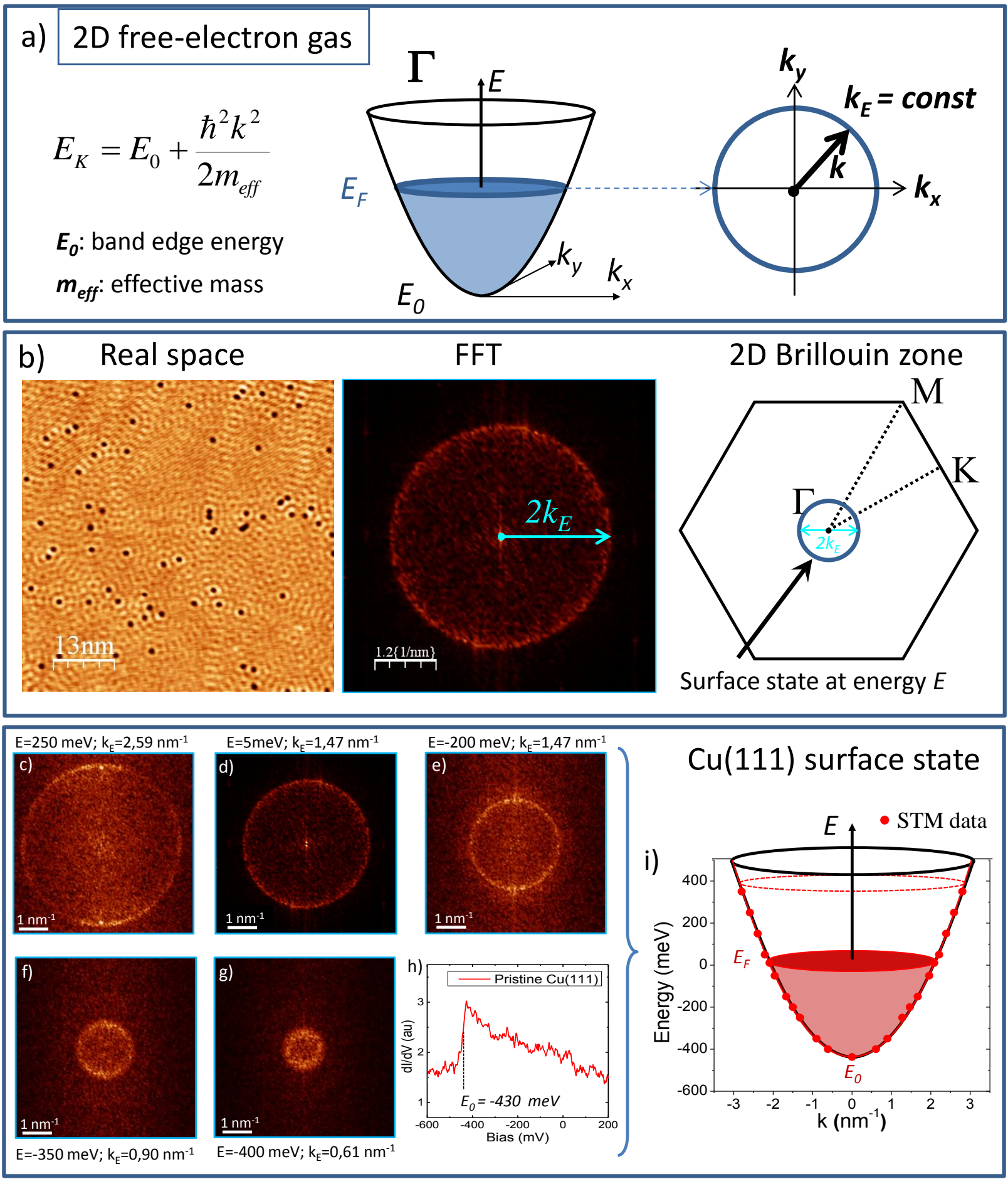

Figure S3. Characterization of 2D electronic states by STM/STS: a) 2D free-electron gas with its characteristic parabolic dispersion and the corresponding 2D constant energy contours. b) Left panel: STM dI/dV map where the standing waves are visible. Central panel: 2D-FT of the left image where a central ring of radius $2 \mathrm{k}$ can be observed. Right panel: $2 \mathrm{D}$ Brillouin zone of $\mathrm{Cu}(111)$. c-g) 2D-FT of $\mathrm{d} / \mathrm{dV}$ maps measured at different energies. $\mathrm{h}$ ) $\mathrm{dI} / \mathrm{dV}$ curve measured on clean $\mathrm{Cu}(111)$ where we can observe the sudden step related with the band edge of the surface state. i) Parabolic dispersion extracted from $\mathrm{dI} / \mathrm{dV}$ maps and $\mathrm{dI} / \mathrm{dV}$ curves as the ones shown in Figures S3c-h. 


\section{Large view of STM/STS data.}

In this Note, to better appreciate the experimental data, we have included some large views of the raw STM data shown in Figure 2 of the manuscript.
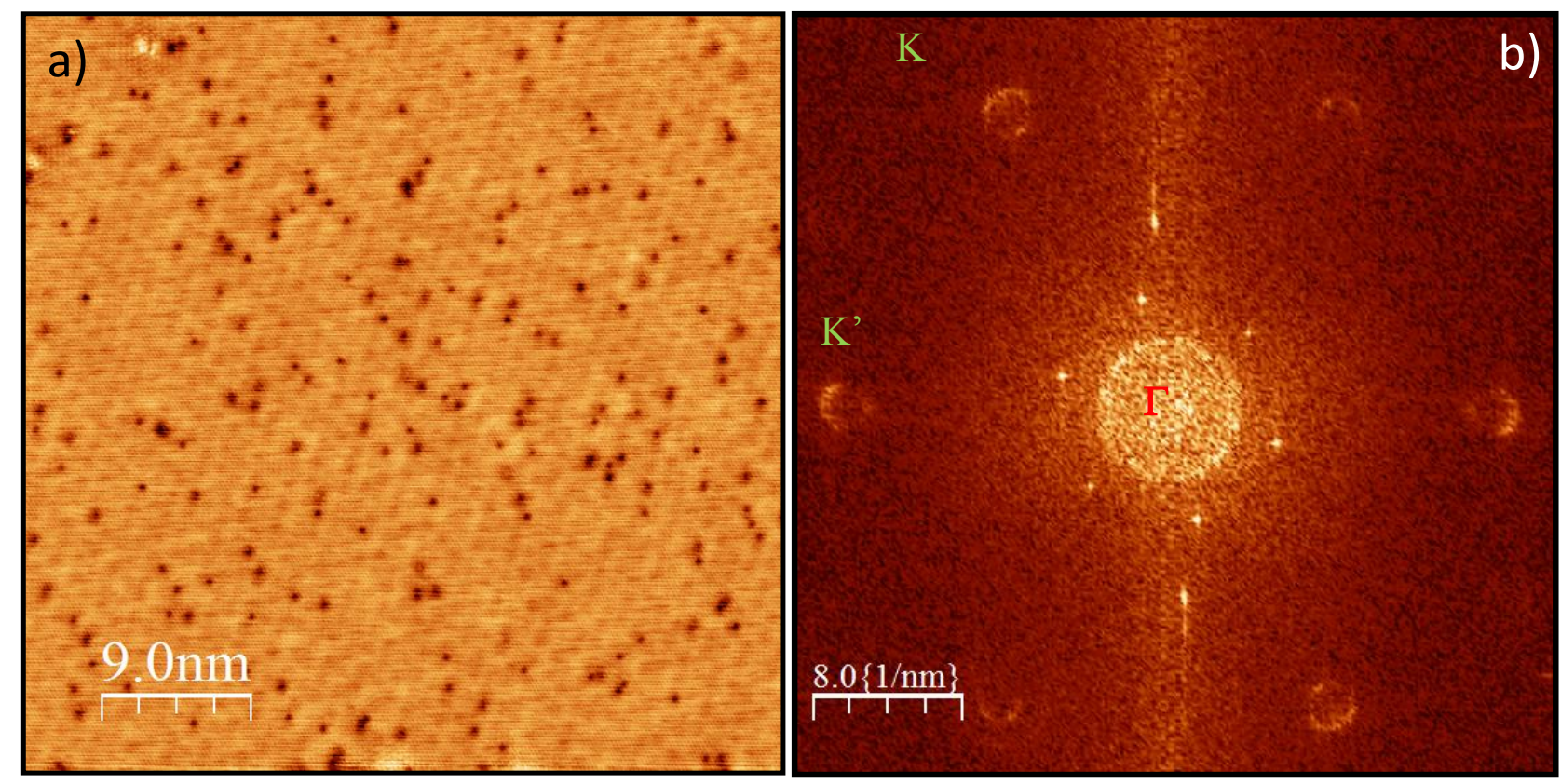

Figure S4. a) Real space STM image of the data shown in Figure $2 b$ of the main manuscript. b) Corresponding 2D-FT (same figure as in Figure 2b). Image size: $45 \times 45 \mathrm{~nm}^{2}$, Tunneling bias: $10 \mathrm{mV}$; tunneling current: $0.47 \mathrm{nA}$

The dispersion of both the graphene Dirac-like band and the $\mathrm{Cu}(111)$ surface state can be obtained from the 2D-FT in energy dependent $d I / d V$ maps, see Figures $2 \mathrm{f}$ and $2 \mathrm{~g}$ respectively. As shown in Figures S5b-c., the radius of the ring like features centered at $K$ and $K^{\prime}$, originated from the intervalley scattering of graphene quasiparticles, shows a strong energy dependence, from which it is possible to determine accurately the dispersion of the graphene $\pi$ bands. A linear dispersion characterized by $k_{F G}=0.54 \mathrm{~nm}^{-1}$ and $v_{F G}=1.1 \cdot 10^{-6} \mathrm{~m} / \mathrm{s}$ is obtained for graphene in the present case, see Figure 2f, Figure S5a and Table 2. To accurately examine the modification of the $\mathrm{Cu}(111)$ surface state by the graphene overlayer, in Figure S5 we plot a superposition of $d I / d V$ curves and 2D-FT maps taken from clean and graphene-covered areas. $d I / d V$ curves measured at positions far away from any surface defect, show an upwards shift of $+0.13 \mathrm{eV}$ of the onset of $\mathrm{Cu}(111)$ surface state band, as determined from the energy shift in the sudden step existing in those curves ( Figure $\mathrm{S} 5 \mathrm{~d})$. Aside from this energy shift, the energy dispersion obtained from the 2D-FT of $d I / d V$ maps shows a non-rigid upwards displacement of the $\mathrm{Cu}(111)$ surface state band, with an effective mass increase from $0.39 m_{e}$ to $0.43 m_{e}$ (Figure $2 \mathrm{~g}$ and Table 1). In Figure S5f we superimpose the central region of the 2D-FT of $d I / d V$ maps measured at $E_{F}$ on covered and uncovered $\mathrm{Cu}(111)$ regions, see also Figure S6 with data plotted separately. A smaller ring, corresponding to a smaller Fermi wavevector in graphene covered areas, indicates the electron depletion of the $\mathrm{Cu}(111)$ surface state band. 
Graphene on $\mathrm{Cu}(111)$

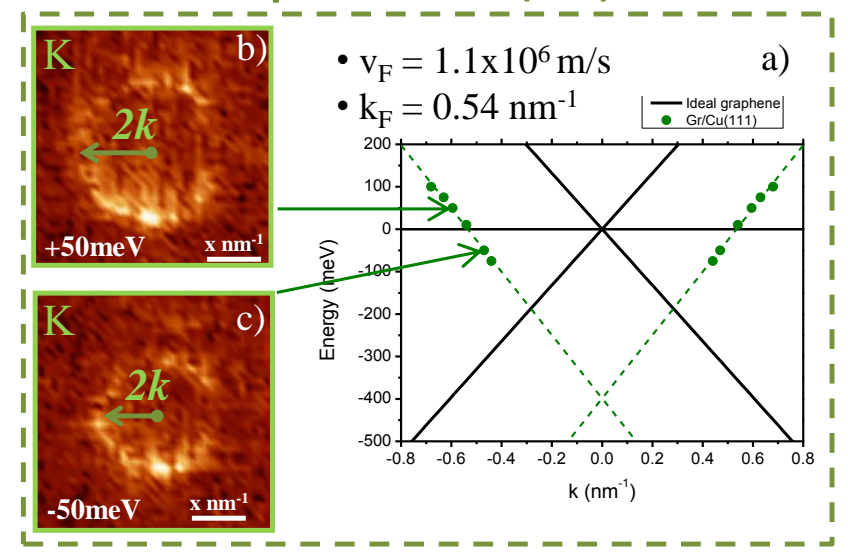

Cu(111) below graphene vs Cu(111)

d)

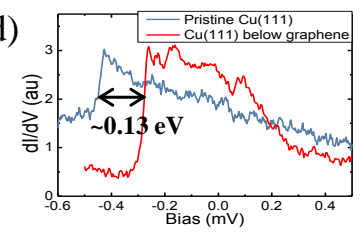

e)

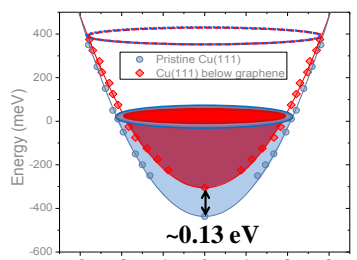

Figure S5. Electronic structure of the $\mathbf{G r} / \mathbf{C u}(111)$ system by LT_STM. Left panel corresponds to the STM experimental electronic characterization of graphene on $\mathrm{Cu}(111)$. The energy dispersion of graphene $\pi$ bands (a) is obtained from the energy dependence of the radius of the ring like features centered at $\bar{K}$ points of the Brillouin zone (b,c). The right panel shows the comparison between the STM experimental electronic properties of pristine $\mathrm{Cu}(111)$ (blue data) and $\mathrm{Cu}(111)$ below graphene (red data):d) $d I / d V$ spectra showing the energy shift of the surface state band edge, e) Energy dispersion of the Shockley surface state, and f) Central region of two 2DFT of $d I / d V$ maps measured at $E_{F}$ showing the different $k_{F C u}$, for pristine $\mathrm{Cu}(111)$ and $\mathrm{Cu}(111)$ below graphene), see also Figure S6.
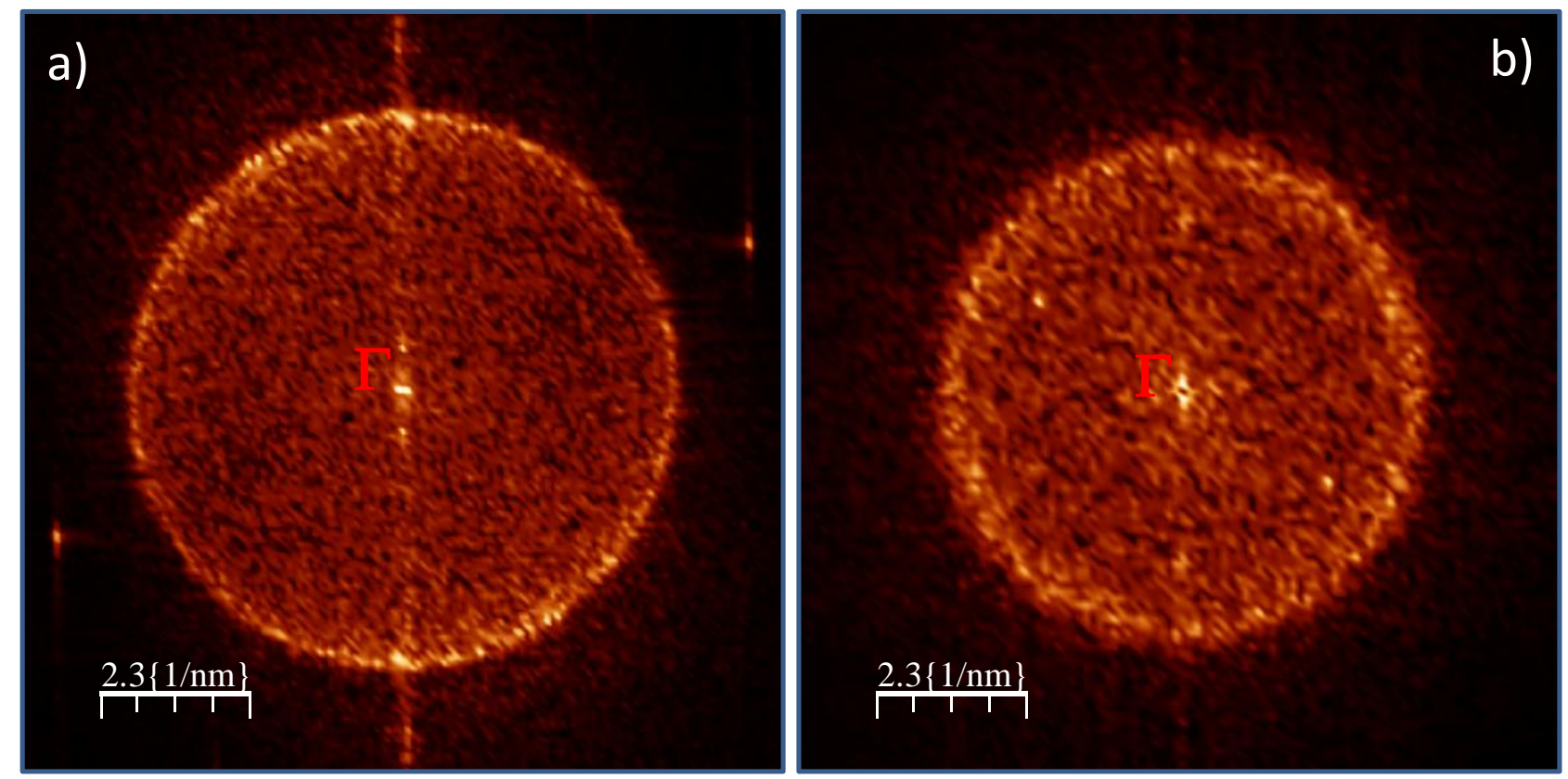

Figure S6. Same data as in Figure S5f plotted separately. a,b) Central region of the 2D-FT of $d I / d V$ maps measured at $E_{F}\left(V_{s}=5 \mathrm{meV}\right)$ on pristine $\mathrm{Cu}(111)$ (a) and graphene covered $\mathrm{Cu}(111)$ regions (b). 


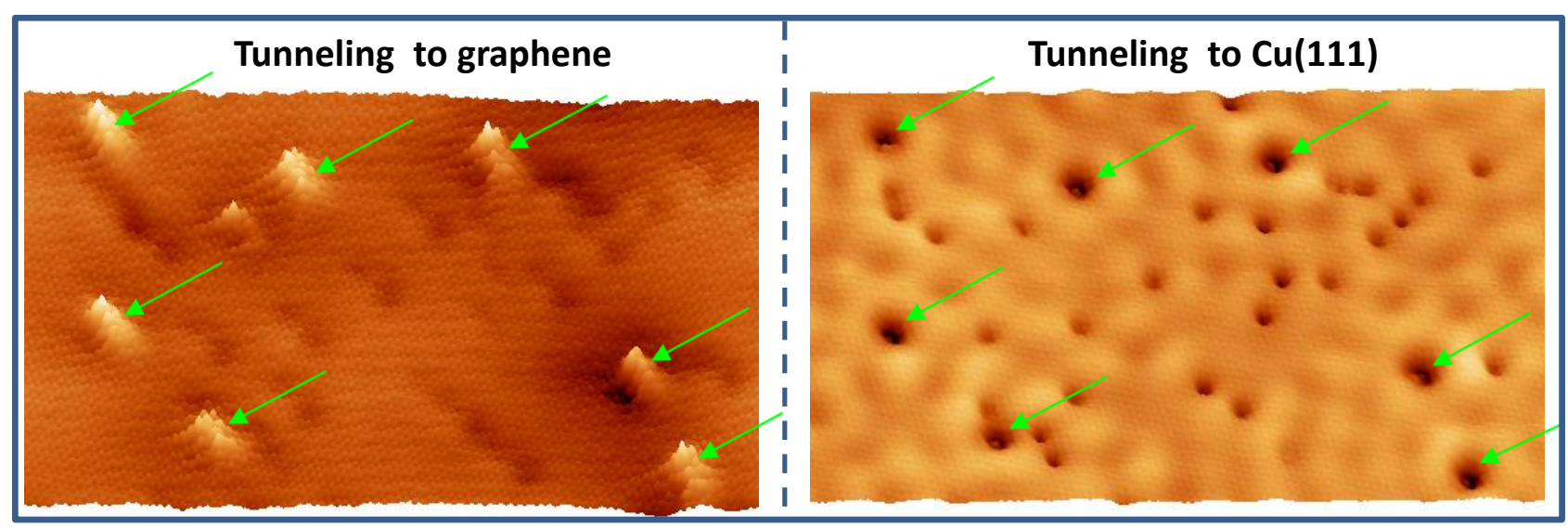

Figure S7. Use of the tunable transparency to follow the interaction between graphene $\mathbf{C}$ vacancies and the $\mathrm{Cu}(111)$ substrate underneath. a) $17 x 10 \mathrm{~nm}^{2} \mathrm{STM}$ image showing a G/Cu(111) sample region with several $\mathrm{C}$-outlined by green arrows- deliberately introduced on the graphene layer by Ar irradiation $(I=0.5 n A)$. b) Same sample region measured at a lower current $(I=0.1 n A)$ to probe the underlying $\mathrm{Cu}(111)$ surface. $\mathrm{C}$ vacancies in graphene act as scattering centers for the $\mathrm{Cu}(111)$ surface quasiparticles generating standing waves below them. 


\section{STM/STS measurements of the graphene electron doping.}

The doping levels are estimated from the change in the Fermi wave-vectors of the graphene and $\mathrm{Cu}(111)$ surface state by comparing values from the pristine systems and the coupled $\mathrm{G} / \mathrm{Cu}(111)$ one.

For graphene: $n=2 \cdot 2 \cdot k_{F f i n}{ }^{2} / 4 \pi=k_{F f i n}{ }^{2} / \pi=+1.0 \cdot 10^{-3} \mathrm{e}^{2} \AA^{2}$;

where $k_{F f i n}=0.55 \mathrm{~nm}^{-1}$ is the Fermi wave-vector of graphene in contact with the $\mathrm{Cu}(111)$ surface.

For $\mathrm{Cu}(111): \mathrm{n}=2 \cdot\left[\mathrm{k}_{\mathrm{Ffin}}{ }^{2}-\mathrm{k}_{\mathrm{Fini}}{ }^{2}\right] / 4 \pi ;=\left[\mathrm{k}_{\mathrm{Ffin}}{ }^{2}-\mathrm{k}_{\mathrm{Fini}}{ }^{2}\right] / 2 \pi=-1.9 \cdot 10^{-3} e / \AA^{2}$;

where $k_{F f i n}=1.80 \mathrm{~nm}^{-1}$ is the Fermi wave-vector of $\mathrm{Cu}(111)$ below the graphene layer and $k_{\text {Fini }}=$ $2.10 \mathrm{~nm}^{-1}$ is the Fermi wave-vector of pristine $\mathrm{Cu}(111)$.

Graphene Graphene on $\mathrm{Cu}(111)$

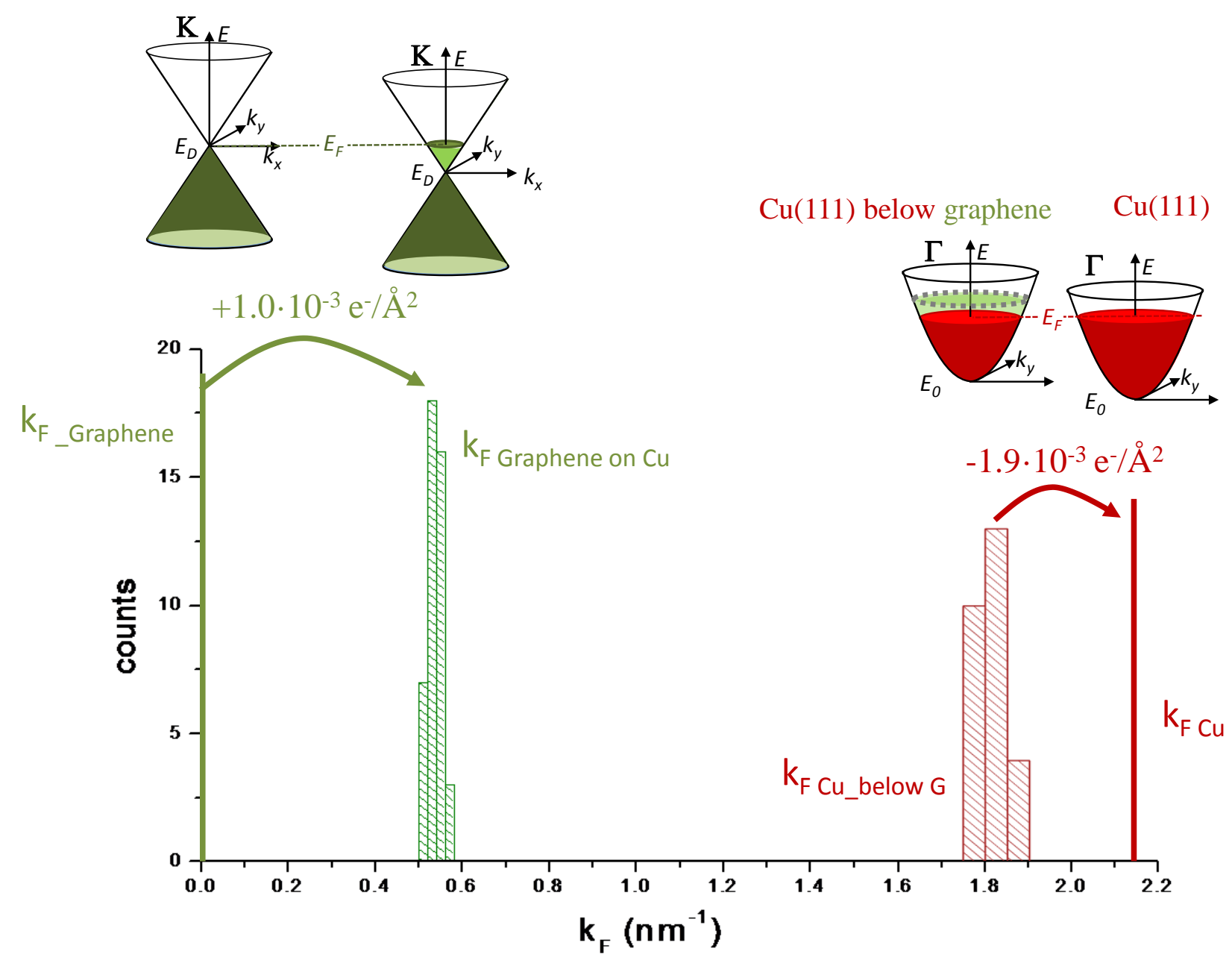

Figure S8. STM/STS measurements over more than 50 different graphene/Cu(111) regions. The lower part of the image shows the histograms for the the STM values obtained from over 50 graphene and $\mathrm{Cu}(111)$ regions exhibiting different orientations. Practically identical doping levels values are found. Such constant experimental doping for the graphene-Cu(111) interface suggests that graphene doping is dominated by the nature of the underlying substrate with little influence of the specific arrangement of the graphene layer. The schematic band dispersions of the upper part, qualitatively illustrate the outcome of our measurements 


\section{Theoretical description of the dispersive (van der Waals) interactions.}

The search for an accurate description of the dispersive interactions --commonly known as van der Waals (vdW)--, is currently a very active field of research. The combination of the PBE exchangecorrelation functional and the Grimme D2 approximation (PBE-D2) is the mostly widely used approach to describe systems where the vdW interactions play an important role. Furthermore, this approach correctly describes the graphene-metal distance in other weakly interacting systems like G/Pt (distance $\sim 3.3 \AA)^{8}$ where this structural parameter has been determined experimentally. For these reasons, we started our study with the PBE-D2 approximation. The large shifts with respect to the experiments in the electronic properties of the $\mathrm{G} / \mathrm{Cu}$ system (position of the Dirac point, modification of the $\mathrm{Cu}$ surface state) when $\mathrm{G}$ is placed at the PBE-D2 energy minimum ( $2.9 \AA$ distance from the $\mathrm{Cu}$ ) clearly show the limitations of the D2 description for the G-Cu system. It is not clear if the overestimation of the interaction, that brings graphene $0.4 \AA$ closer in $\mathrm{Cu}$ than in other weakly interacting metals, comes from the C6 coefficients or the radii for the cutoff function, and more theoretical effort is needed in this direction. Nevertheless, the important conclusion achieved from the comparison of theory and experiment is that the G-metal coupling is correctly described by PBE at the $3.4 \AA \mathrm{G}-\mathrm{Cu}$ distance. More accurate approaches for the van der Waals interactions point out the same direction, in particular calculations with a more sophisticated semiempirical approach PBE-D3 ${ }^{9}$-where the C6 coefficients depend on the chemical environment--, and the vdW-inclusive functional (optB86b-vdW) proposed by Klimes et al ${ }^{10}$. This functional is a modified version of the non-local vdW-density functional by Dion et al. ${ }^{11}$. The optB86b-vdW functional provides a good agreement between experimental adsorption energies and geometries and calculated values for benzene on several transition metal surfaces, a system considered as a benchmark ${ }^{12}$. PBE-D3 and optB86b-vdW give G-Cu distance of $\sim 3.2 \AA$ and $\sim 3.3 \AA$ respectively, approaching the $3.4 \AA$ distance determined from the characterization of the electronic properties. This shows the potential of our combined experimental-theoretical approach to test and tune the new approximations for the description of the vdW interactions currently under development ${ }^{13}$. 


\section{Charge transfer and level alignment.}

The n-doping of the $\mathrm{G}$ on $\mathrm{Cu}$ has been explained in terms of both the difference of $\mathrm{G}$ and $\mathrm{Cu}$ work functions and the interaction between them ${ }^{14}$. Graphene workfunction, $4.6 \mathrm{eV}$ in our calculations (see Figure $\mathrm{S} 9 \mathrm{~b}$ ), is $\sim 0.2 \mathrm{eV}$ smaller than the $\mathrm{Cu}(111)$ one, $\sim 4.8 \mathrm{eV}$, this implies that a dipole with its positive pole places on the $\mathrm{G}$ area should be induced. On Figure S9a we show the differential average-plane electronic charge density calculated for the $1 \times 1 \mathrm{G} / \mathrm{Cu}(111)$ slab at a $3.4 \AA \mathrm{G}-\mathrm{Cu}$ separation, the dipole located on the G-Cu coupling area is clearly observed with a charge $q=1.4 x$ $10^{-3} e^{-} / \AA^{2}$. This dipole yields a $\mathrm{G} / \mathrm{Cu}(111)$ work function of $4.3 \mathrm{eV}$. Although the differential charge density shows that on the graphene area exits a deficit of electronic charge, the graphene bands are shifted towards negative energies, $n$-doping the $G$ layer. This downward shift is produced by the G$\mathrm{Cu}$ coupling: the non-local van der Waals forces attach $\mathrm{G}$ to $\mathrm{Cu}$ at distances where there is a significant overlapping between the $\pi$-graphene states and the $\mathrm{Cu}$ electrons clearly observed on Figure $4 \mathrm{a}$ in the main text.

The combination of our STM experiments and calculations shows that the $G$ bands are overpopulated with $1.0 \cdot 10^{-3} \mathrm{e} / \AA^{2}$ and the $\mathrm{Cu}$ surface state loses $1.9 \cdot 10^{-3} \mathrm{e}^{\circ} \AA^{2}$, while, at the same time, there is a total dipole with its positive charge of $q=1.4 \times 10^{-3} e^{-} / \AA^{2}$ around the G layer (highlighted with a blue background in Figure S9a). Although interaction produces not only charge transfer but also complex charge redistribution, we can explain this apparent contradiction with a simple picture: graphene bands receive electrons through the $\mathrm{G}-\mathrm{Cu}$ coupling, this charge comes from the dispersed $\mathrm{Cu}$ surface state that shows significant weight on the area where the $\mathrm{G}$ layer is located. In fact in order to produce in that area a deficiency of electrons the $\mathrm{Cu}$ surface state loses more charge that the collected by the G. The rest of the charge lost by the Shockley state goes to the negatively charged area around the $\mathrm{Cu}$ surface (highlighted as red background in Figure S9a) to complete the dipole.
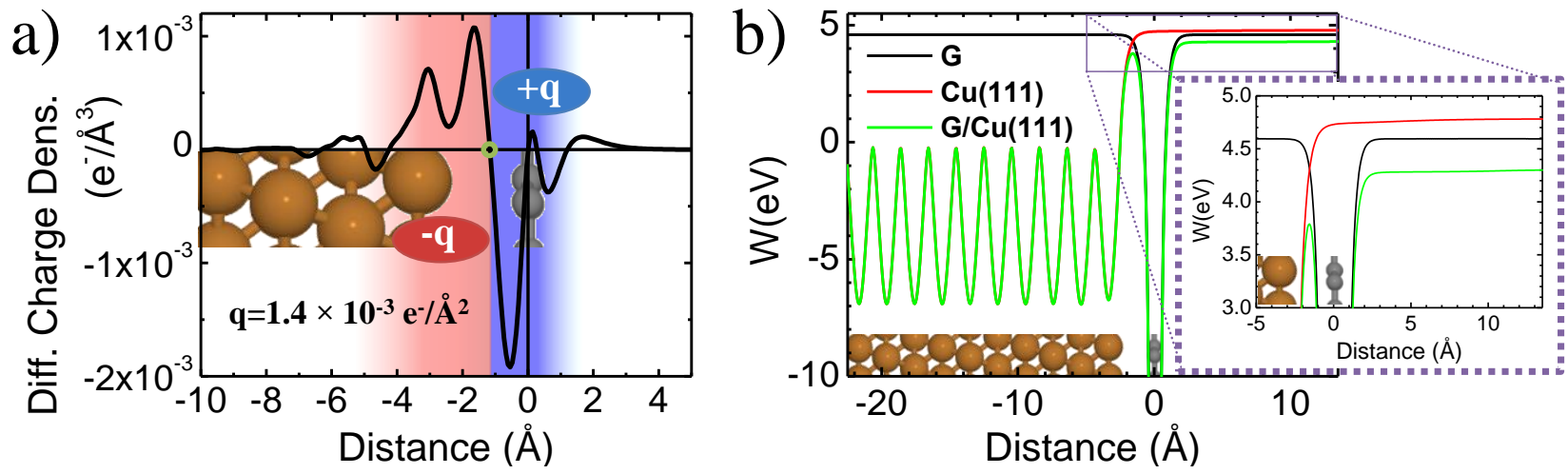

Figure S9. Charge density distribution and level alignment on G/Cu(111). a) Differential average plane charge density calculated as the difference between the charge density of the $\mathrm{G} / \mathrm{Cu}$ system and the isolated $\mathrm{G}$ and $\mathrm{Cu}$ slabs. A dipole pointing out from $\mathrm{Cu}$ to $\mathrm{G}$ localized between the metal surface and the layer is clearly observed (red and blue areas show respectively excess and deficit of electron charge density). The charge, q, integrated from the nodal point (green circle) to the infinity is $1.4 \times 10^{-3} e^{-} / \AA^{2}\left(\sim 1 / 2\right.$ of the dipole charge prevoisuly reported $\left.{ }^{14}\right)$. The formation of this dipole results on a reduction of the workfunction of the $\mathrm{G} / \mathrm{Cu}$ respect of the $\mathrm{G}$ and $\mathrm{Cu}$ ones. $\mathrm{b}$ ) Electrostatic potential of $\mathrm{G}$ (black line), $\mathrm{Cu}(111)$ (red line) and $\mathrm{G} / \mathrm{Cu}(111)$ (green line) respect to their corresponding Fermi levels. The asymptotic value (4.6 eV, $4.8 \mathrm{eV}$ and $4.3 \mathrm{eV}$ for $\mathrm{G}, \mathrm{Cu}$, and $\mathrm{G} / \mathrm{Cu}$ respectively) is the workfunction provided by the PBE calculation. 


\section{Simulation results of the $\sqrt{57} \times \sqrt{ } \sqrt{57}$ Moiré pattern.}

The theoretical results presented on the main text have been calculated with a small $1 \times 1$ surface unit cell. Due to the lattice mismatch, imposing a $1 \times 1$ periodicity implies a strong deformation of either the $\mathrm{G}$ layer or the metal. We have used the $\mathrm{Cu}$ lattice parameter to correctly describe the surface state, thus the $\mathrm{G}$ layer has been stretched by $4.6 \%$. This induced strain is so high, with such a large energy penalty that makes the $1 \times 1$ moire unrealistic. This is consistent with the fact that the $\mathrm{G} / \mathrm{Cu}$ moiré patterns observed in the experiments are much larger. We have carried out simulations with a $\sqrt{57 x} \sqrt{57}$ moiré with respect to the $\mathrm{G}$, one of the smallest observed experimentally, but with only 4 $\mathrm{Cu}$ layers (see Figure S10). Although this calculation cannot provide a proper description of the influence of $\mathrm{G}$ on the $\mathrm{Cu}$ surface state (the simulation with $15 \mathrm{Cu}$ layers is beyond the current computing capabilities), it does shed light on the possible influence of strain in the results discussed above. In this moiré, the lattice mismatch is very small, only $0.3 \%$, so the uncertainties coming from the choice of the lattice parameter are minimized. We have found an average G-Cu distance of $3.0 \AA$ A, slightly larger than in the $1 \times 1$ case, and a topographic corrugation of the $\mathrm{G}$ layer of $9 \mathrm{pm}$. The areas with $\mathrm{C}$ atoms on top of the $\mathrm{Cu}$ atoms are the lowest ones, while the ones corresponding to hollow positions are the highest (see Figure S10b). Similarly to the $1 \mathrm{x} 1 \mathrm{case}$, this moire also shows a large downward shift of the Dirac point $(0.6 \mathrm{eV})$, indicative again of a large G-Cu interaction. Furthermore, the Dirac point position coincides with the case of the $1 \times 1$ surface cell for the same G$\mathrm{Cu}$ distance (see Figure 3d on the main text). These results show that the link between G-metal distances and energy shifts are essentially the same for the real $\sqrt{57 x} \sqrt{57}$ moiré pattern and the $1 \times 1$ surface cell, and, thus, validate our previous discussion of the G-metal interaction on the properties of both graphene and the $\mathrm{Cu}$ surface state.
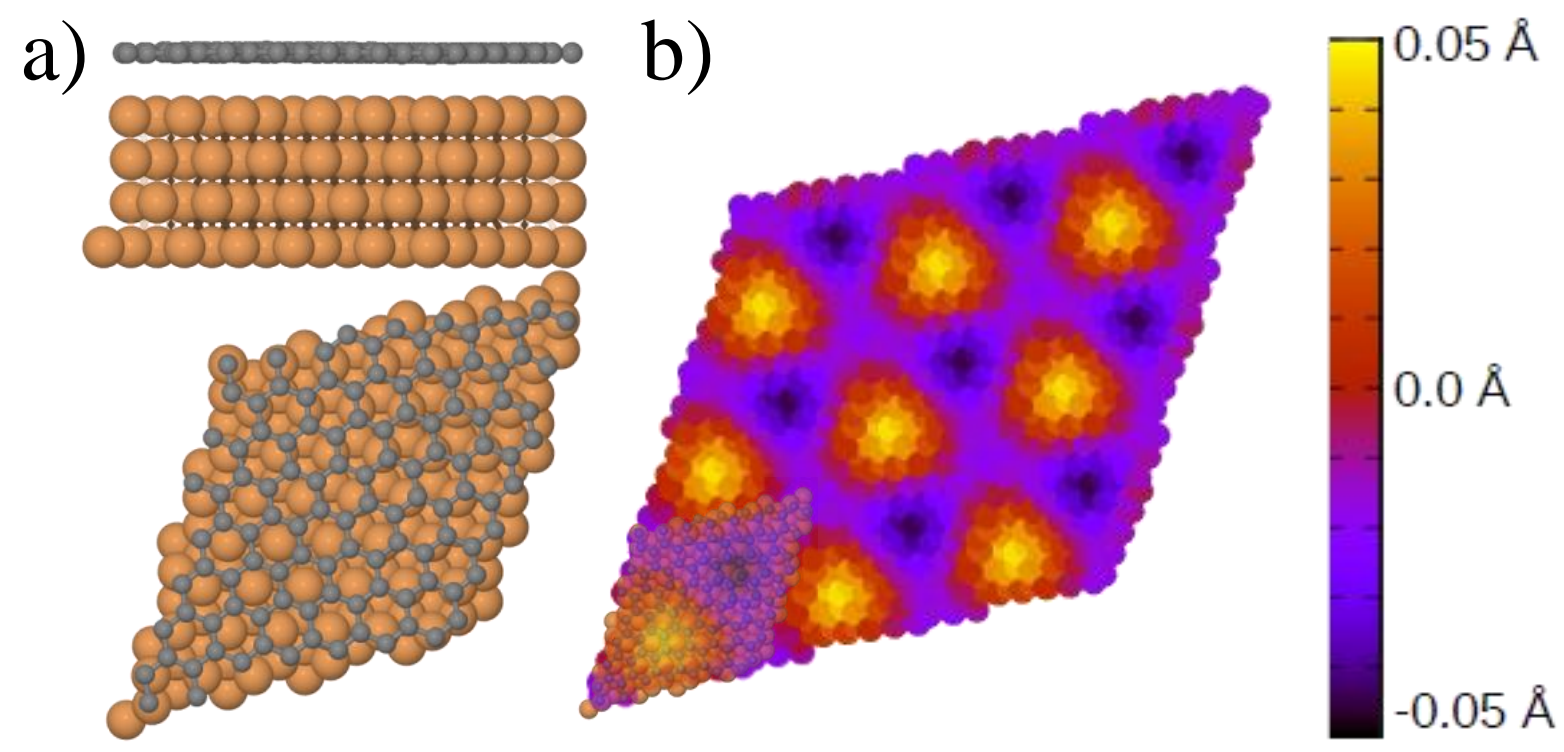

Figure S10. The $\sqrt{57} \times \sqrt{57}$ moiré pattern. a) Ball-and-stick model of the atomic structure of $\mathrm{G} / \mathrm{Cu}(111)$ forming a $\sqrt{5} 7 \mathrm{x} \sqrt{57}$ moiré pattern (respect to the $\mathrm{G}$ ) calculated with PBE+D2. The average G-Cu distance is $3 \AA$. b) Color map of the corrugation of the G layer for his moiré pattern. Area with smaller G-Cu distance corresponds to $\mathrm{C}$ atoms stacking on the top of $\mathrm{Cu}$ atoms, while maximum separation corresponds to carbons staking on hollow positions. The layer is very flat with a corrugation of just $9 \mathrm{pm}$. 


\section{Further details of the explanation of graphene tunable transparency.}

The decay of the electronic states depends, in principle, on the energy range but, in the case of the $\mathrm{G}$ on $\mathrm{Cu}$, our calculations show that our main conclusion (the slow out-of-plane decay of the $\mathrm{Cu}$ surface state with respect to the $\mathrm{G}$ states) holds for a broad energy range. We illustrate this point with figure S11 that shows the projected charge density calculated for an energy window from -2 $\mathrm{eV}$ to $\mathrm{E}_{\mathrm{F}}$.

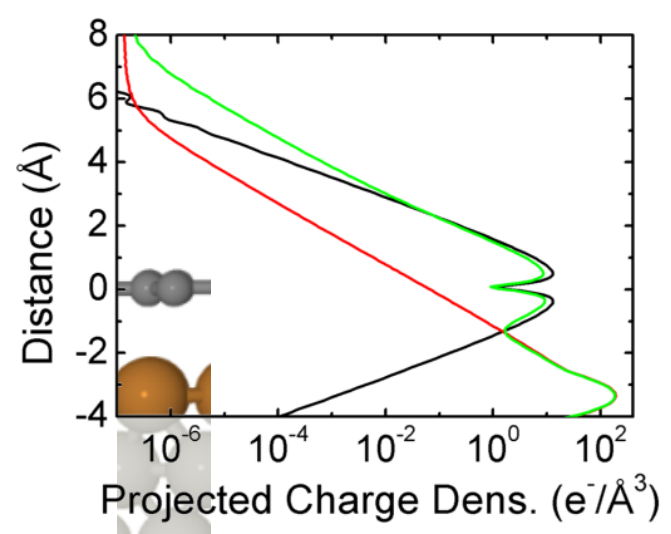

Figure S11. Projected charge density on the energy range from $-2 \mathrm{eV}$ to $E_{F}$ integrated in the xy plane as function of perpendicular position for graphene on $\mathrm{Cu}$ (green line), for a $\mathrm{Cu}(111)$ slab (red line) and for an isolated graphene (black line). Note that all curves share the same relative positions, being graphene its distance origin. We observe that the $\mathrm{Cu}$ surface state decay length is larger than graphene, so for graphene on $\mathrm{Cu}$, the charge at larger distances is dominated by $\mathrm{Cu}$.

a)

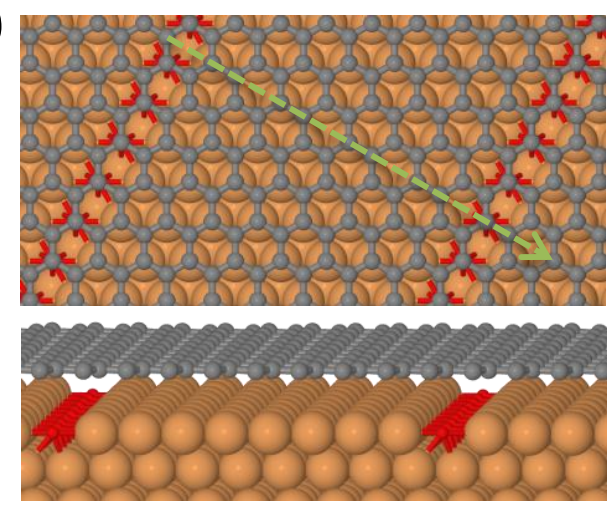

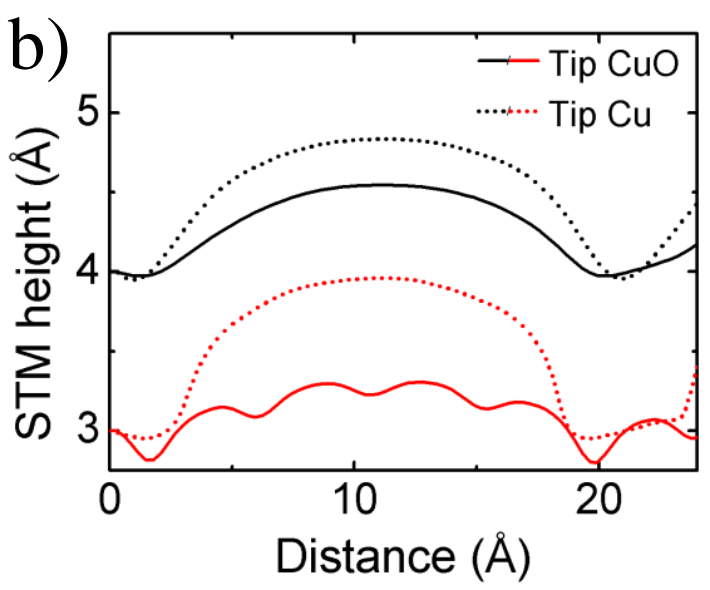

Figure S12 Comparison between the constant height profiles calculated with the purely metallic Cu10 tip (dotted lines) and the contaminated $\mathrm{CuO}$ tip (solid lines). a) Stick-and-ball model of the atomic configuration of a graphene layer on a $\mathrm{Cu}$ surface tailored with an oxygen row substituting $\mathrm{Cu}$ surface atoms in order to create a spatial modulation of the $\mathrm{Cu}$ surface state (see Methods). b) Simulated constant current STM profiles along the green line marked on a) for different tip sample separations and at a $-0.1 \mathrm{~V}$ bias (the STM height is defined respect to the $\mathrm{z}$ coordinate of the carbon atom, at distance $=0 \AA$.). At large distances $(\mathrm{d}>4 \AA$ ), both tips are sensing the charge modulations originated in the $\mathrm{Cu}$ surface states by defects on its surface. At closer distances ( $d=3 \AA$ ), the contrast of the Cu tip is still dominated by the metal states, while there are already clear features that reveal atomic contrast in $\mathrm{G}$. 


\section{Tip state identification from $\mathrm{dI} / \mathrm{dV}$ spectra.}

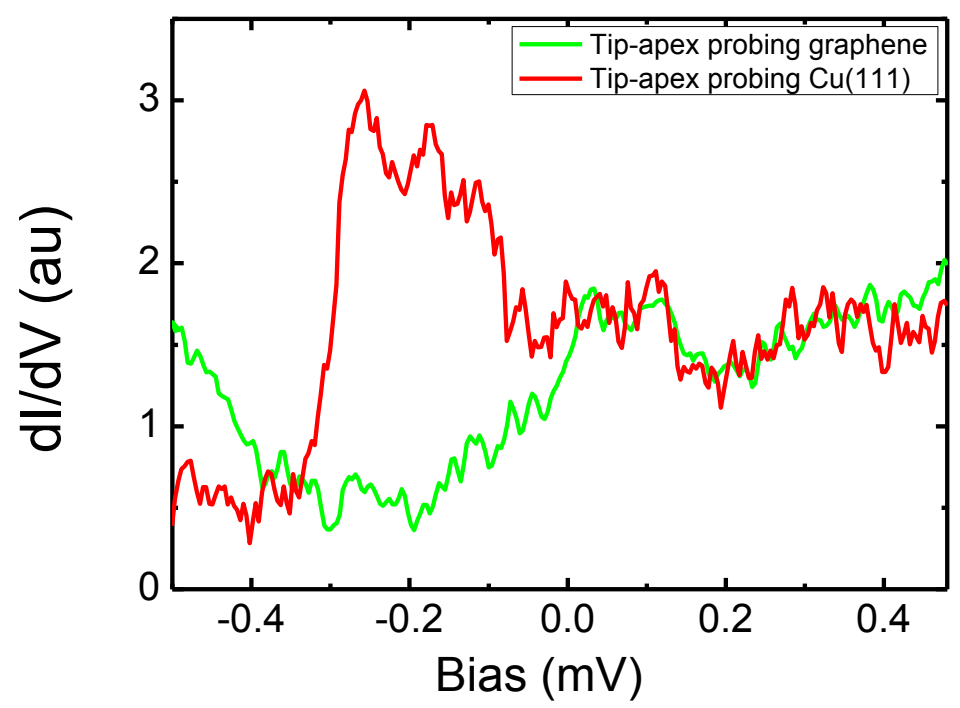

Figure S13. Tip state identification from dI/dV spectra. $d I / d V$ curves measured on the graphene/ $\mathrm{Cu}(111)$ sample region shown in Figure 1 with different tip apex terminations to selectively probe graphene (green curve) or the $\mathrm{Cu}(111)$ surface states (red curve). Green/red curve was obtained with the same tip apex termination than Figure $1 \mathrm{a}-\mathrm{b}$. Both $\mathrm{dI} / \mathrm{dV}$ curves were measured with the same tunneling parameters: nominal junction impedance of $1.2 \mathrm{G} \Omega(-500 \mathrm{mV}$, $0.4 \mathrm{nA}$ ). Changes in the tip visualization mode were deliberately introduced by controlled indentations of the STM tip onto the substrate, see Methods section for details.

\section{Experimental parameters for selectively removing $\mathbf{H}$ atoms.}

Choosing the appropriate tunneling parameters atomic $\mathrm{H}$ can be removed from graphene with atomic precision. The role played by the STM tip is to selectively modify the binding energy landscape of $\mathrm{H}$ atoms to produce the required $\mathrm{H}$ manipulation. To selectively remove $\mathrm{H}$ atoms from the graphene sample we approached the STM tip towards the sample. This can be done by continuously increasing, under feedback control, the setpoint tunneling current on top of the selected $\mathrm{H}$ atom until it is desorbed, or by switching the feedback off and slightly decreasing the tipsample distance on top of it. It is also possible to completely remove all $\mathrm{H}$ atoms from a graphene region by imaging it at high currents. The precise tunneling values for the manipulation depend on each specific tip apex, but for the same tip apex those values are very reproducible. As a rough guide, for removing $\mathrm{H}$ atoms it is usually enough to approach the STM tip by 2-3 $\AA$ (opening the feedback loop at a nominal junction impedance of $20 \mathrm{M} \Omega ; V_{\text {bias }}=0.01 \mathrm{~V}, I_{t}=0.5 \mathrm{nA}$ ). 


\section{Supporting references.}

Martinez-Galera, A. J.; Brihuega, I.; and Gomez-Rodriguez, J. M. Ethylene Irradiation: A New Route to Grow Graphene on Low Reactivity Metals. Nano. Lett. 11 (9), 3576 (2011).

Crommie, M. F.; Lutz, C. P.; and Eigler, D. M. Imaging Standing Waves in a 2-Dimensional Electron-Gas. Nature 363 (6429), 524 (1993).

Hasegawa, Y.; Avouris, P. Direct Observation of Standing-Wave Formation at Surface Steps Using Scanning Tunneling Spectroscopy. Phys. Rev. Lett. 71 (7), 1071 (1993).

Petersen, L.; Sprunger, P. T.; Hofmann, Ph.; Lægsgaard, E. B.; Briner, G.; Doering, M.; Rust, H.P.; Bradshaw, A. M.; Besenbacher, F.; Plummer, E. W. Direct imaging of the twodimensional Fermi contour: Fourier-transform STM. Phys. Rev. B 57 (12), R6858 (1998).

Sprunger, P. T.; Petersen, L.; Plummer, E. W.; Lægsgaard, E.; Besenbacher, F. Giant friedel oscillations on the beryllium(0001) surface. Science 275 (5307), 1764 (1997).

Hörmandinger, G. Imaging of the $\mathrm{Cu}(111)$ Surface-State in Scanning-TunnelingMicroscopy. Phys. Rev. B 49 (19), 13897 (1994).

Reinert, F.; Nicolay, G.; Schmidt, S.; Ehm, D.; Hüfner S. Direct measurements of the L-gap surface states on the (111) face of noble metals by photoelectron spectroscopy. Phys. Rev. B 63 (11), 115415 (2001).

Ugeda, M. M.; Fernández-Torre, D.; Brihuega, I.; Pou, P.; Martínez-Galera, A. J.; Pérez, R.; Gómez-Rodríguez, J. M. Point Defects on Graphene on Metals. Phys. Rev. Lett. 107 (11) (2011).

Grimme, S.; Antony, J.; Ehrlich, S.; Krieg, H. A consistent and accurate ab initio parametrization of density functional dispersion correction (DFT-D) for the 94 elements HPu.J. Chem. Phys. 132 (15), 19 (2010).

Klimes, J.; Bowler, D. R.; Michaelides, A. Van der Waals density functionals applied to solids. Phys. Rev. B 83 (19), 13 (2011).

Dion, M.; Rydberg, H.; Schröder, E.; Langreth, D. C.; Lundqvist, B. I. Van der Waals density functional for general geometries. Phys. Rev. Lett. 92 (24), 246401 (2004).

Carrasco, J.; Liu, W.; Michaelides, A.; Tkatchenko, A. Insight into the description of van der Waals forces for benzene adsorption on transition metal (111) surfaces.J. Chem. Phys. 140 (8) (2014).

Tkatchenko, A.; Current Understanding of Van der Waals Effects in Realistic Materials. Adv. Funct. Mater. 25, 2054 (2015).

Khomyakov, P. A.; Giovannetti, G.; Rusu, P. C.; Brocks, G.; van den Brink, J.; Kelly, P. J. First-principles study of the interaction and charge transfer between graphene and metals. Phys. Rev. B 79 (19) (2009). 
